\title{
Heme oxygenase- $I$ is a predictive biomarker for therapeutic targeting of advanced clear cell renal cell carcinoma treated with sorafenib or sunitinib
}

This article was published in the following Dove Press journal:

OncoTargets and Therapy

10 August 2015

Number of times this article has been viewed

\author{
Wan-Xiang Zheng* \\ Fei Yan* \\ Qin Xue \\ Guo-Jun Wu \\ Wei-Jun Qin \\ Fu-Li Wang \\ Jun Qin \\ Chun-Juan Tian \\ Jian-Lin Yuan
}

Department of Urology, Xijing Hospital, Fourth Military Medical University, Xi'an, Shaanxi, People's Republic of China

*These authors contributed equally to this work
Correspondence: Jian-Lin Yuan

Department of Urology, Xijing Hospital, Fourth Military Medical University,

No 127, Changle West Road, Xi'an,

Shaanxi, 7I0032, People's Republic of

China

Tel +86 I36 09|2 3|55

Fax +862984775317

Email jianliny 1010@। 26.com
Background: We analyzed the expression of heme oxygenase-1 (HO-1) in patients undergoing radical nephrectomy for advanced clear cell renal cell carcinoma (CC-RCC) and evaluated the effects of the targeted therapies treated with sorafenib and sunitinib.

Methods: Expression of HO-1 in cancer tissue from 66 patients was measured by immunohistochemical staining. The patients received either oral sorafenib $(n=40)$ or oral sunitinib $(n=26)$ within 4 weeks after nephrectomy and were followed up long term to determine the tumor response and prognosis.

Results: Our current study revealed a high HO-1 expression level in 57.6\% (38/66) of patients and a low HO-1 expression level in 42.4\% (28/66) of patients with CC-RCC. The study also revealed that patients with high $\mathrm{HO}-1$ expression did not have a higher objective response rate ( $2.6 \%$ versus $53.6 \%, P<0.01)$, clinical benefit rate $(47.4 \%$ versus $92.9 \%, P<0.01)$, longer progression-free survival ( 4.4 versus 42 months, $P=0.022)$, or overall survival $\left(\chi^{2}=4.775\right.$, $P=0.029)$ than patients with low HO-1 expression. In the low HO-1 level group, a higher tumor response rate and a longer survival time was achieved in patients who received sorafenib or sunitinib. Multivariate analysis showed that HO-1 expression was an independent prognostic factor for tumor response and overall survival.

Conclusion: High expression of HO-1 was associated with a lower tumor response rate and a shorter overall survival time when compared with low expression of HO-1. Overall, HO-1 expression might be a useful biomarker for predicting the response to sunitinib and sorafenib for patients with metastatic CC-RCC.

Keywords: HO-1, renal cell carcinoma, sunitinib, sorafenib target therapy

\section{Introduction}

Renal cell carcinoma (RCC) is a common urological malignancy, accounting for approximately $3 \%-4 \%$ of all human malignancies. ${ }^{1,2}$ Clear cell RCC (CC-RCC) is the most common pathology of RCC. ${ }^{3}$ Surgical resection is the preferred method for treating early renal cancer; however, because of insidious onset and lack of an appropriate early diagnosis index, $30 \%$ of the patients with RCC are not eligible for surgery at the time of initial diagnosis because of the metastasis. ${ }^{4}$ Because of the high rate of recurrence and metastasis of CC-RCC, historically there were no reasonable and effective treatment options for patients with advanced RCC.

After thorough research of the RCC signaling pathways, several biologic agents that target the vascular endothelial growth factor (VEGF) pathway began to attract the attention of researchers. ${ }^{1,2-5}$ Sorafenib and sunitinib are multitargeted tyrosine kinase inhibitors that target several tyrosine kinases, including vascular endothelial growth factor (VEGFR), platelet-derived growth factor receptor- $\beta$ (PDGFR), RAF-1, wild-type and mutant BRAF, 
CSF-1R, and so on. ${ }^{2,6-8}$ The antitumor efficacy of sorafenib and sunitinib has been demonstrated in both preclinical and clinical studies, indicating their potential to significantly improve progression-free survival (PFS) and overall survival (OS). ${ }^{6,8,9}$ However, not all patients respond to these agents, and their expense is often a financial burdens to patients. The primary challenge with targeted medical therapy is how to select patients who are most likely to respond to a specific agent. Identifying markers that predict the efficacy of targeted therapy on CC-RCC would allow for more individualized treatment options. ${ }^{10}$

Heme oxygenase-1 (HO-1) is a stress-inducible molecule that has anti-oxidative injury and anti-apoptotic properties that play a cytoprotective role. ${ }^{11}$ However, HO-1 also protects cancer cells, which plays an important role in promoting tumor growth. Many studies have shown that HO-1 expression is often increased in various tumor tissues, including melanoma, ${ }^{12}$ pancreatic cancer, ${ }^{13}$ liver cancer, ${ }^{14}$ and RCC. ${ }^{15,16}$ Considering the roles of HO-1 in the development, invasion, and metastasis of tumors, it might be a potential target of cancer therapy. ${ }^{17}$ In this study, we analyzed the expression of HO-1 in patients undergoing radical nephrectomy for advanced CC-RCC and evaluated the effects of targeted therapy treated with sorafenib or sunitinib. To our knowledge, the present study is the first to assess the possibility that HO- 1 could be a therapeutic target to predict the efficacy of sorafenib and sunitinib in advanced CC-RCC.

\section{Materials and methods}

\section{Patients and samples}

Advanced CC-RCC specimens $(n=66)$ were collected from patients who underwent surgical resection in the Department of Urology of Xijing Hospital from June 2006 to May 2014. Patients were selected according to the following criteria: age $\geq 18$ years; advanced metastatic CC-RCC confirmed by post-operative pathology; distant metastasis developed before the operation; nephrectomy performed without prior systemic treatment or molecular targeted therapy; presence of Response Evaluation Criteria In Solid Tumors (RECIST) ${ }^{18}$ measurable lesions; Eastern Cooperative Oncology Group (ECOG) performance status of $\leq 2$; life expectancy greater than 12 weeks; and received oral sorafenib or sunitinib as the first-line therapy after nephrectomy. The protocol to obtain tissue samples was performed with the approval and oversight of the Xijing Hospital Ethics Committee. Written informed consent was obtained from all patients involved.

\section{Histopathological data}

Within 4 weeks after radical nephrectomy, 40 patients received oral sorafenib and 26 patients received oral sunitinib.
The sorafenib dosing was $400 \mathrm{mg}$ orally twice daily over a 4-week cycle, and the sunitinib dosing was $50 \mathrm{mg}$ orally once daily for 4 weeks followed by 2 weeks off (a 6-week cycle), and continued until disease progression or occurrence of intolerable adverse reactions. All patients were followed up long term for 5.1-67.5 months, with an average of 22.1 months. Patients were initially assessed within 4 weeks after nephrectomy to determine the efficacy and safety of the targeted therapy, and were then assessed every 3-4 weeks. The follow-up deadline was November 1, 2014.

\section{Immunohistochemical staining}

To investigate the expression of HO-1 in CC-RCC, fresh nephrectomy specimens were fixed in 4\% paraformaldehyde overnight and were then embedded in paraffin. Paraffin sections ( $4 \mu \mathrm{m}$ thick) were deparafinized in xylene, rehydrated in serial alcohol solutions of decreasing dilution, and were then treated with $3 \% \mathrm{H}_{2} \mathrm{O}_{2}$ to block endogenous peroxidase activity. Antigen retrieval was done by immersing the slides in citrate buffer and microwaving them for 10 minutes. Slides were incubated with $10 \%$ goat serum in phosphate-buffered saline (PBS) for 30 minutes to block nonspecific staining. The sections were then incubated with a primary anti-HO-1 monoclonal antibody (ab-13248, Abcam, Cambridge, UK) at 1:100. The slides were then washed by PBS and incubated with horseradish peroxidase-labeled anti-mouse immunoglobulin $\mathrm{G}(\mathrm{IgG})(\mathrm{H}+\mathrm{L})$ for 2 hours at room temperature. Staining was revealed using diaminobenzidine (DAB) and counter-stained with hematoxylin.

The staining results were observed by microscope to facilitate statistical analysis of HO-1 expression, and the average optical density was determined using five high-magnification fields for each section by using Image Pro Plus.

\section{Evaluation of targeted treatment effect}

Target lesion selection and tumor response assessment were performed according to the RECIST criteria. ${ }^{18}$ The therapeutic effect was assessed after patients took the drug for 3 months. Tumor response was assessed based on four perspectives: 1) complete response (CR), 2) partial response (PR), 3) stable disease (SD), and 4) progressive disease (PD). The baseline comparisons were the computed tomography (CT) or magnetic resonance imaging (MRI) before the treatment. If the patients were determined to have CR or PR, the evaluation was repeated after 4 weeks; if the patients were determined to have $\mathrm{SD}$, the evaluation was repeated after 2 months. Based on the tumor response statistics, we calculated the objective response rate (ORR) and clinical benefit rate (CBR) of sorafenib and sunitinib. 
Prognosis was assessed according to PFS and OS. PFS was measured from treatment initiation to the first documented clinical progression. The OS was calculated from treatment initiation to the date of death.

\section{Statistical analysis}

All statistical analyses were performed using SPSS Statistics Version 19.0 (IBM Corporation, Armonk, NY, USA). A $P$-value of $<0.05$ was considered statistically significant. Pearson $\chi^{2}$ was used to compare the percentage difference between the two groups. To compare the differences between CR, PR, SD, and PD, normality and homogeneity of variance tests were used. Quantitative data were analyzed using one-way analysis of variance. When non-normality in a distribution was identified, medians were calculated. According to the distribution of $\mathrm{CR}, \mathrm{PR}, \mathrm{SD}$, and $\mathrm{PD}$, the critical value of high or low HO-1 expression was assessed by the receiver operating characteristic curve (ROC curve) and Youden index. The PFS and OS were estimated using the Kaplan-Meier method, and the log-rank test was used to calculate the differences between the curves. Multivariate logistic analysis was carried out to identify predictors of drug sensitivity. A Cox proportional hazards regression model was used to estimate the prognostic significance of each variable, and the hazard ratio (HR) of $>1.0$ represents a shorter duration for patients with high $\mathrm{HO}-1$-expressing tumors.

\section{Results}

\section{Expression of HO-I in CC-RCC tissues}

HO-1 was mainly expressed in the cytoplasm of the tumor cells (Figure 1). The optimal value of the average optical density was evaluated by the ROC curve and Youden index to measure the expression level of HO-1. The sensitivity and specificity of this cutoff value in predicting tumor $\mathrm{CR} / \mathrm{PR}$ and $\mathrm{SD} / \mathrm{PD}$ was $74.0 \%$ and $93.7 \%$, respectively. We then defined high HO-1 expression as a value of $>0.087$, and low HO- 1 expression as a value of $<0.087$. In our results, $57.6 \%$ (38/66) of CC-RCC patients had high HO-1 expression, and $42.4 \%(28 / 66)$ patients had low expression.

\section{Correlation between $\mathrm{HO}-\mathrm{I}$ expression and clinicopathological features}

We assessed the pathological features of the RCC tumors according to the HO-1status of the tumor (Table 1). The results showed that the expression level of HO-1 in CC-RCC patients was not associated with clinicopathological features, including age, sex, T stage, Fuhrman nuclear grading and distant metastasis, Memorial Sloan-Kettering Cancer Center (MSKCC) prognostic risk factors, and Heng prognostic risk factors.

\section{Tumor response assessment for sunitinib and sorafenib}

Based on the RECIST criteria, ${ }^{18}$ the response to sunitinib or sorafenib was assessed in 66 patients after the drug was taken for 3 months (Table 2). At the time of analysis, two CR, nine PR, $15 \mathrm{SD}$, and $14 \mathrm{PD}$ were achieved in the sorafenib group and zero CR, five PR, $13 \mathrm{SD}$, and eight PD were achieved in sunitinib group. The ORR and CBR in patients treated with sorafenib was $27.5 \%(11 / 40)$ and $65.0 \%$ (26/40), respectively, and in the sunitinib group, the ORR and CBR was $19.2 \%(5 / 26)$ and $69.2 \%(18 / 26)$, respectively.
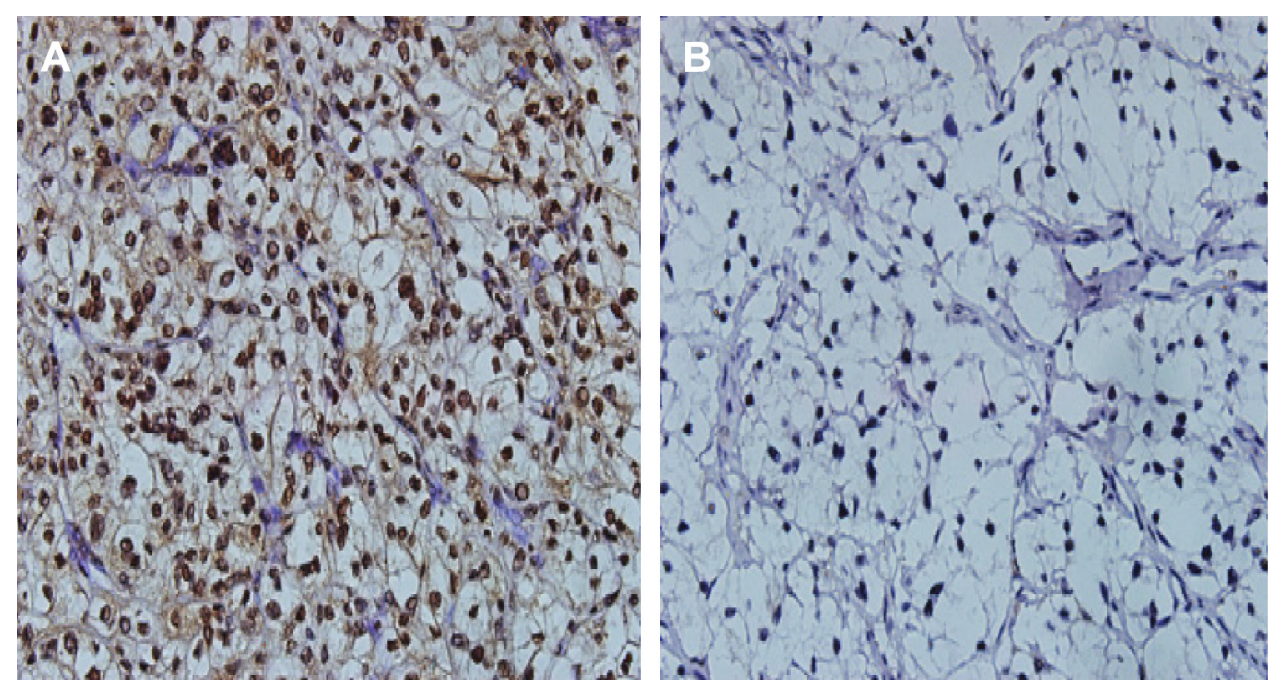

Figure I Representative images of immunoreactivity for $\mathrm{HO}-\mathrm{I}$ in tumor tissues.

Notes: (A) Positive expression; (B) negative expression (magnification, 400×).

Abbreviation: HO-I, heme oxygenase- I. 
Table I Relationship between HO-I expression and the pathological parameters of CC-RCC

\begin{tabular}{|c|c|c|c|c|c|}
\hline \multirow[t]{2}{*}{ Clinical parameters } & \multirow[t]{2}{*}{ Classification } & \multirow[t]{2}{*}{ Number of patients } & \multicolumn{2}{|c|}{ HO-I expression } & \multirow[t]{2}{*}{$P$-value } \\
\hline & & & $\operatorname{Low}(n)$ & High (n) & \\
\hline \multirow[t]{2}{*}{ Sex } & Male & 53 & 23 & 30 & 0.747 \\
\hline & Female & 13 & 5 & 8 & \\
\hline \multirow[t]{2}{*}{ Age } & $<60$ & 38 & 15 & 23 & 0.572 \\
\hline & $\geq 60$ & 28 & 13 & 15 & \\
\hline \multirow[t]{2}{*}{ T stage } & $\mathrm{I}-2$ & 51 & 20 & 31 & 0.331 \\
\hline & 3 and 4 & 15 & 8 & 7 & \\
\hline \multirow[t]{2}{*}{ Fuhrman grade } & I and II & 21 & 9 & 12 & 0.961 \\
\hline & III and IV & 45 & 19 & 26 & \\
\hline \multirow[t]{3}{*}{ Metastatic organ } & Lung & 39 & 18 & 21 & $0.74 I$ \\
\hline & Multiple organs & 10 & 4 & 6 & \\
\hline & Other & 17 & 6 & 11 & \\
\hline \multirow[t]{3}{*}{ MSKCC risk groups } & Favorable & 14 & 9 & 5 & 0.092 \\
\hline & Intermediate & 39 & 16 & 23 & \\
\hline & Poor & 13 & 3 & 10 & \\
\hline \multirow[t]{3}{*}{ Heng-risk groups } & Favorable & 8 & 6 & 2 & 0.080 \\
\hline & Intermediate & 40 & 17 & 23 & \\
\hline & Poor & 18 & 5 & 13 & \\
\hline
\end{tabular}

Abbreviations: HO-I, heme oxygenase-I; CC-RCC, clear cell renal cell carcinoma; MSKCC, Memorial Sloan-Kettering Cancer Center; n, number of patients.

There were no statistical differences of ORR $\left(\chi^{2}=0.587\right.$; $P=0.444)$ and CBR $\left(\chi^{2}=0.127 ; P=0.722\right)$ between the sorafenib and sunitinib groups. Based on these findings, we determined there was approximately equal sensitivity of CC-RCC to sunitinib and to sorafenib.

To test whether HO-1 expression has a predictive value for the targets of different agents, we firstly investigated the association of HO-1 status with the sensitivity of patients who received sorafenib or sunitinib. The results are shown in Table 2.

The ORR was $61.1 \%$ versus $0 \%$ for sorafenib-treated patients with low versus high tumor HO-1 expression, compared with $40 \%$ versus $6.3 \%$ for sunitinib-treated patients. Meanwhile, for both sorafenib- or sunitinib-treated patients, the CBR of low HO-1 expression was significantly higher than the CBR of high HO-1 expression. The results showed that the HO-1 expression level is significantly associated with sensitivity to sorafenib and sunitinib. Multivariate logistic analysis showed that HO-1 expression $(P=0.000)$ and MSKCC risk groups $(P=0.027)$ were independent predictors of targeted therapy sensitivity (Table 3 ).

\section{Survival analysis}

The prognostic effects in CC-RCC were analyzed by Cox regression analysis. At the time of analysis, the median PFS of the 40 patients treated with sorafenib was 14.63 months (95\% CI, 9.23-20.03 months), and the median PFS of the 26 patients treated with sunitinib was not reached. The $P$-value of the log-rank test was 0.093 , which showed that the PFS of the sunitinib group was higher than the sorafenib group. Then, we further evaluated the relationship of HO-1 and PFS. There were 28 (42.4\%) and 38 (57.6\%) patients classified into the low HO-1 level group and high HO-1 level group, respectively. Median PFS for the low HO-1 group was 42.0 months, whereas those patients with a high HO-1 level had a PFS of 4.4 months $\left(\chi^{2}=5.212 ; P=0.022\right)$ (Figure 2A).

Table 2 Sensitivity for sorafenib- and sunitinib-treated patients according to tumor HO-I staining status

\begin{tabular}{|c|c|c|c|c|}
\hline & \multicolumn{2}{|l|}{ Sorafenib } & \multicolumn{2}{|l|}{ Sunitinib } \\
\hline & Low HO-I & High HO-I & Low HO-I & High HO-I \\
\hline Number of patients & 18 & 22 & 10 & 16 \\
\hline Complete response $(n)$ & 2 & 0 & 0 & 0 \\
\hline Partial response (n) & 9 & 0 & 4 & 1 \\
\hline Progressive disease $(n)$ & 2 & 12 & 0 & 8 \\
\hline Stable disease $(n)$ & 5 & 10 & 6 & 7 \\
\hline ORR (CR + PR) & $61.1 \%$ & 0 & $40 \%$ & $6.3 \%$ \\
\hline $\mathrm{CBR}(\mathrm{CR}+\mathrm{PR}+\mathrm{SD})$ & $88.9 \%$ & $45.5 \%$ & $100 \%$ & $50 \%$ \\
\hline
\end{tabular}

Abbreviations: CR, complete response; PR, partial response; PD, progressive disease; SD, stable disease; HO-I, heme oxygenase-I; ORR, objective response rate; $\mathrm{CBR}$, clinical benefit rate; $\mathrm{n}$, number of patients. 
Table 3 Multivariate logistic analysis to identify predictors of targeted therapy sensitivity

\begin{tabular}{llll}
\hline Covariates & OR & $\mathbf{9 5 \% ~ C l}$ & $\boldsymbol{P}$-value \\
\hline HO-I expression (high versus low) & 20.328 & $5.114-80.802$ & 0.000 \\
$\begin{array}{l}\text { MSKCC risk groups (poor versus } \\
\text { intermediate versus favorable) }\end{array}$ & 3.445 & $1.150-10.319$ & 0.027 \\
$\begin{array}{l}\text { Heng risk groups (poor versus } \\
\text { intermediate versus favorable) }\end{array}$ & 0.807 & $0.27 \mathrm{I}-2.406$ & 0.700 \\
\hline
\end{tabular}

Abbreviations: OR, odds ratio; $\mathrm{Cl}$, confidence interval; $\mathrm{HO}-\mathrm{I}$, heme oxygenase-I; MSKCC, Memorial Sloan-Kettering Cancer Center.

The present results suggest that CC-RCC patients with a high HO-1 level demonstrated a significantly shorter PFS than those with a low HO-1 level $(\mathrm{HR}=2.272 ; 95 \% \mathrm{CI}$, $1.102-4.686 ; P=0.026)$. We also investigated the potential role of HO-1 in predicting the PFS from sorafenib and sunitinib treatment. The results are shown in Figure 2B and C. In brief, the median PFS of patients who received sorafenib was 16.1 and 4.3 months in the low and high HO-1 level groups (HR=1.629; 95\% CI, 0.731-3.626; $P=0.233$ ), respectively. The median PFS of patients with a low HO-1 level who received sunitinib was longer than the median PFS in the high HO-1 level group $(\mathrm{HR}=58.09$; 95\% CI, 0.261-12939.780; $P=0.141$; and $\chi^{2}=7.791 ; P=0.005$, respectively).

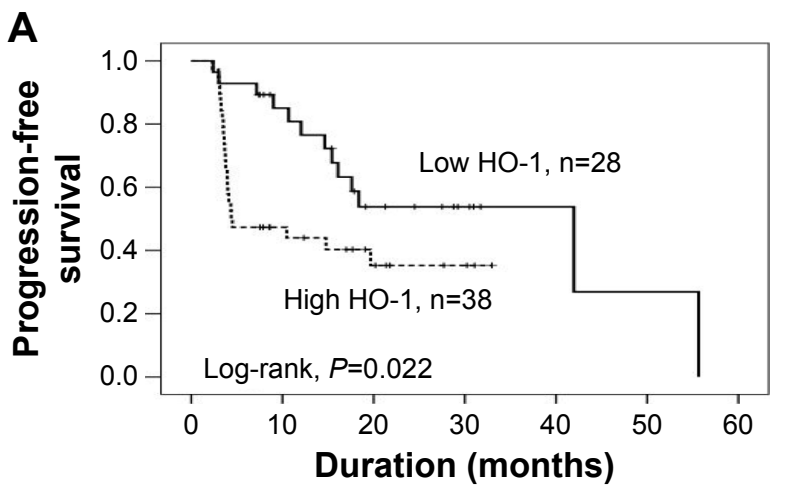

At the time of data analysis, the median OS had not been reached in the sunitinib group, and the median OS was 48.97 months among patients treated with sorafenib; the difference was statistically significant (log-rank test, $\chi^{2}=4.467$; $P=0.035)$. Then, we further evaluated the relationship of $\mathrm{HO}-1$ and OS. The median OS in patients with a high expression of HO-1 was significantly shorter than in patients with a low expression of HO-1 (HR=3.425; 95\% CI, 1.069-10.973 $P=0.038$; and $\chi^{2}=4.775 ; P=0.029$, respectively) (Figure 3A). The same results were achieved in the patients who were treated with sorafenib $(\mathrm{HR}=3.826,95 \% \mathrm{CI}, 1.148-12.749$; $P=0.029$; and $\left.\chi^{2}=5.395, P=0.020\right)$ or sunitinib $(\mathrm{HR}=41.165$, 95\% CI, 0.000-6937271.379; $P=0.545$; and $\chi^{2}=1.122$; $P=0.290$, respectively), that is to say, in the high HO-1 group, regardless of the treatment provided, the patients had a shorter OS compared with the low HO-1 group (Figure 3B and C).

We also conducted a multivariate analysis to identify the correlation between the clinicopathological parameters and PFS or OS of patients. The results suggest that age was a predictor of PFS (Table 4), and that HO-1 expression and Heng prognostic risk groups were independent prognostic factors of OS (Table 5).

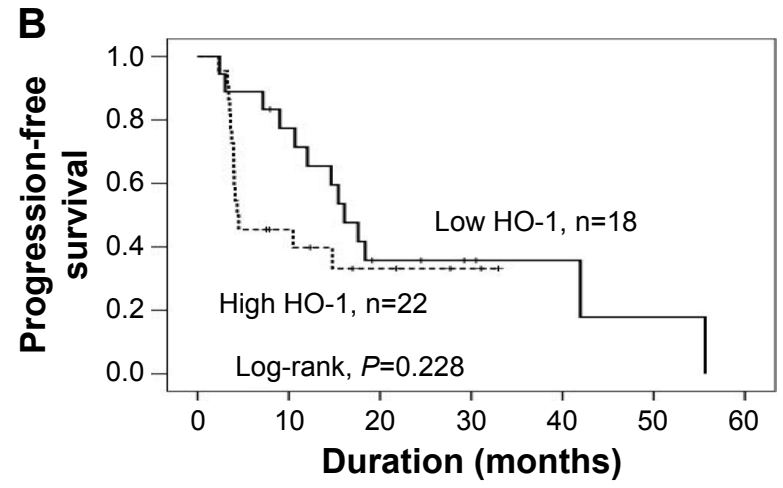

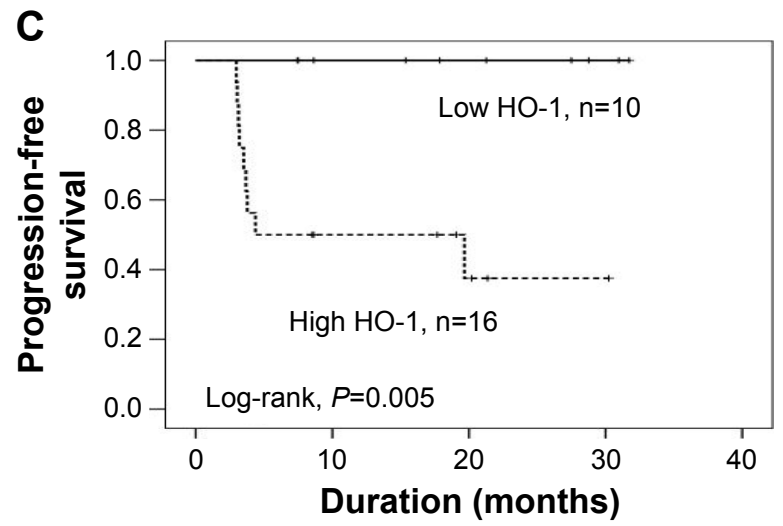

Figure 2 Kaplan-Meier PFS curves of CC-RCC patients with different levels of HO-I expression.

Notes: (A) All patients; (B) sorafenib group; and (C) sunitinib group.

Abbreviations: PFS, progression-free survival; CC-RCC, clear cell renal cell carcinoma; HO-I, heme oxygenase-I; n, number of patients. 
A

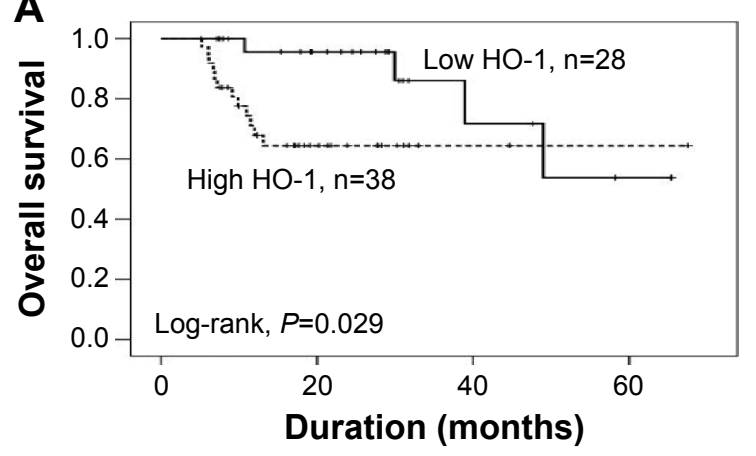

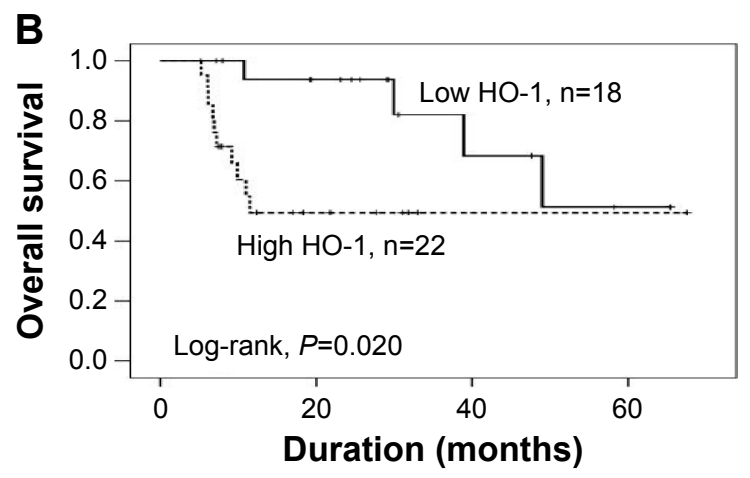

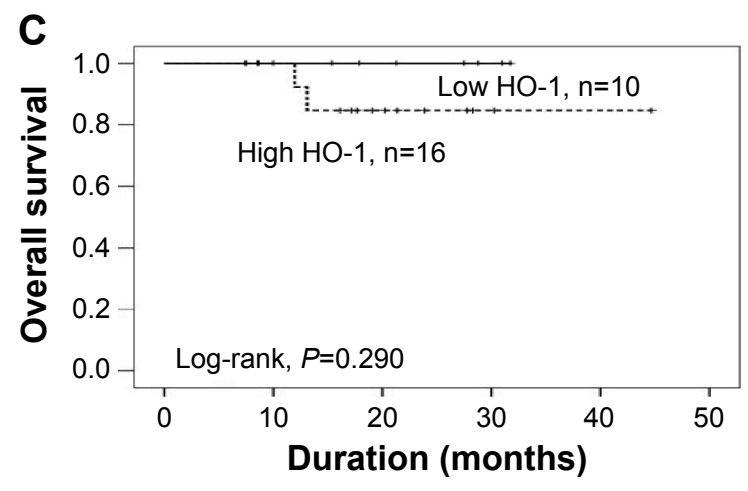

Figure 3 Kaplan-Meier OS curves of CC-RCC patients with different levels of HO-I expression.

Notes: (A) All patients; (B) sorafenib group; (C) sunitinib group.

Abbreviations: OS, overall survival; CC-RCC, clear cell renal cell carcinoma; HO-I, heme oxygenase-I; n, number of patients.

\section{Discussion}

Most patients with advanced metastatic RCC have a poor prognosis; most die within 1 year, and only $10 \%$ survive for 5 years. ${ }^{19-21}$ CC-RCC does not usually respond to radiotherapy or chemotherapy, ${ }^{22-24}$ so, over the past 20 years, immunotherapy has been the main treatment option. ${ }^{24}$ High-dose interleukin-2 or interferon alpha were the preferred therapies for advanced RCC, ${ }^{25}$ but the serious side effects and the poor curative effects of the drugs limited their use. ${ }^{26,27}$ In recent years, targeted agents, such as sorafenib and sunitinib, have been used for RCC therapy, and have quickly replaced traditional cytokine therapy as the first-line treatment. However, because of uncertainty about the outcomes of targeted therapy on different patients, including invalidation, drug resistance, recurrence, and metastasis, the exploration of predictive and prognostic biomarkers became increasingly important to improve the selection of targeted agents for patients. In recent years, many prognostic biomarkers have been identified in patients with RCC who were treated with targeted agents, but the markers must be validated prospectively and independently before they can be adopted into clinical use. ${ }^{28}$ Furthermore, assessment of current biomarkers and identification of novel biomarkers might be the most important area of research regarding targeted therapy.

HO-1 is expressed in various human tumors, where it serves as an essential protective molecule by improving tumor survival and resistance to anticancer treatment. ${ }^{29}$ It has been hypothesized that HO-1 might have an important

Table 4 Multivariate analysis of the correlation between the clinicopathological parameters and PFS of patients

\begin{tabular}{llllll}
\hline Covariates & Coefficient & Standard error & HR & 95\% Cl for HR & P-value \\
\hline HO-I expression (high versus low) & 0.657 & 0.391 & 1.930 & $0.897-4.153$ & 0.093 \\
Sex (male versus female) & -0.798 & 0.423 & 0.450 & $0.196-1.033$ & 0.060 \\
Age ( $\geq 60$ versus $<60$ years) & -0.792 & 0.398 & 0.453 & $0.208-0.989$ & 0.047 \\
MSKCC risk groups (poor versus & 0.385 & 0.365 & 1.470 & $0.719-3.005$ \\
intermediate versus favorable) & & & & 0.291 \\
Heng risk groups (poor versus & 0.123 & 0.428 & 1.131 & $0.489-2.617$ \\
intermediate versus favorable) & & & & & 0.774 \\
\hline
\end{tabular}

Abbreviations: PFS, progression-free survival; HR, hazard ratio; Cl, confidence interval; HO-I, heme oxygenase-I; MSKCC, Memorial Sloan-Kettering Cancer Center. 
Table 5 Multivariate analysis of the correlation between the clinicopathological parameters and OS of patients

\begin{tabular}{llllll}
\hline Covariates & Coefficient & Standard error & HR & 95\% Cl for HR & P-value \\
\hline HO-I expression (high versus low) & 1.259 & 0.641 & 3.520 & $1.003-12.359$ & 0.050 \\
Heng risk groups (poor versus & $1.28 \mathrm{I}$ & 0.479 & $3.60 \mathrm{I}$ & $1.409-3.60 \mathrm{I}$ & 0.007 \\
intermediate versus favorable) & & & & & \\
\hline
\end{tabular}

Abbreviations: OS, overall survival; $\mathrm{HR}$, hazard ratio; $\mathrm{Cl}$, confidence interval; $\mathrm{HO}-\mathrm{I}$, heme oxygenase-I.

role in tumorigenesis and drug resistance mechanisms, and might be a potential predictor for targeted therapy.

In the present study, we investigated the clinical efficacy of sorafenib and sunitinib in advanced metastatic CC-RCC, detected the expression of HO-1 in tumor tissues, and then analyzed the correlations between HO- 1 and targeted therapy. Our aim was to study if HO-1 expression could predict the efficacy of sorafenib or sunitinib treatment.

Sorafenib and sunitinib were approved for the treatment of metastatic RCC, which targets the VEGF pathway to inhibit invasion and dissemination of RCC, because they showed significant efficacy. ${ }^{30-34}$ However, up to $30 \%$ of patients might not benefit from these therapies because of various baseline clinical features and tumor characteristics. ${ }^{30-34}$ In our present study, there was no significant difference in tumor response to sorafenib and sunitinib; however, patients treated with sunitinib demonstrated a significantly longer OS time than those with sorafenib. This result was consistent with the fact that sunitinib is recommended as a first-line treatment for cancer by the US Food and Drug Administration (FDA). ${ }^{34}$ Both the median PFS (14.63 months) and the median OS (48.97 months) among patients treated with sorafenib in our study were longer than the PFS and OS of patients treated with sorafenib in Europe and the USA. ${ }^{30}$ This result is consistent with other research indicating that sorafenib had greater efficacy in Chinese patients. ${ }^{35}$

To investigate the expression of HO-1 in CC-RCC in the current study, the cancer specimens and corresponding normal tissues were collected for immunohistochemical staining. The results confirmed that the average optical density value of cancer tissues is much higher than that of corresponding noncancerous tissues. The difference was statistically significant (data not shown), which is consistent with findings in other studies. ${ }^{15,16}$ Then, we divided the HO-1 expression into high and low levels according to the critical value of the average optical density value. High HO-1 expression was found in 38 tumors (57.6\%).

Then, we explored the relationship between high HO-1 expression levels with the clinicopathological parameters. The results suggest that HO-1 expression is not associated with clinical characteristics; therefore, we considered that
HO-1 might have a unique function in CC-RCC. We further investigated the association of HO-1 status with drug efficacy. With regard to tumor response, we demonstrated that patients with high HO-1 expression did not have a higher ORR (2.6\% versus $53.6 \% ; P<0.01$ ). To detect the validity of $\mathrm{HO}-1$ as a predictor, we constructed the ROC curve according to the data of SD/PD (test group) and PR/CR (standard group); the area under the curve was 0.879 , which means moderate accuracy of HO-1 as a predictor. With regard to survival time, high expression of HO-1 was associated with shorter PFS ( 4.4 versus 42 months, $P=0.022$ ) or $\mathrm{OS}\left(\chi^{2}=4.775 ; P=0.029\right)$ when compared with low expression of HO-1. Multivariate logistic analysis showed that HO-1 expression $(P=0.000)$ and MSKCC risk groups $(P=0.027)$ were independent predictors of target therapy sensitivity. Interestingly, multivariate logistic analysis suggested that age was a predictor of PFS; the patients who were aged $\geq 60$ years exhibited a longer PFS, which was presumably because of the decreased tumor growth rates in older adults. HO-1 expression and Heng prognostic risk groups were independent prognostic factors of OS.

Therefore, our results indicated that tumors with a high HO-1 level might represent worse outcomes and require more intensive or targeted treatment strategies. Similarly, for the patients who had received a targeted therapy agent, a high HO-1 level also significantly correlated to poor response and prognosis.

To our knowledge, this was the first study to investigate whether HO-1 is a predictor for CC-RCC response to the targeted therapies sorafenib and sunitinib. The results of the present study demonstrate that HO-1 plays a pivotal role in resistance to targeted therapy. It is important to understand how HO-1 influences the efficacy of targeted therapy; therefore, research on the mechanism of HO-1 was our primary objective.

\section{Conclusion}

In summary, HO-1 might represent a potential novel biomarker for prediction of targeted therapy efficacy in CC-RCC. Determination of the HO-1 expression level in tumors might lead to progress in individualized diagnostic and anti-cancer treatment strategies. 


\section{Acknowledgment}

This work was supported by grants from the translational medicine research projects of Xijing Hospital (grant number XJZT13Z05).

\section{Disclosure}

The authors report no conflicts of interest in this work.

\section{References}

1. Jemal A, Siegel R, Xu J, Ward E. Cancer statistics, 2010. CA Cancer J Clin. 2010;60(5):277-300.

2. Koul H, Huh JS, Rove KO, et al. Molecular aspects of renal cell carcinoma: a review. Am J Cancer Res. 2011;1(2):240-254.

3. Zhao J, Zhu Y, Zhang C, et al. Sorafenib or sunitinib as postoperative adjuvant therapy for Chinese patients with locally advanced clear cell renal cell carcinoma at high risk for disease recurrence. Urol Oncol. 2013;31(8):1800-1805.

4. Coppin C, Porzsolt F, Awa A, Kumpf J, Coldman A, Wilt T. Immunotherapy for advanced renal cell cancer [review]. Cochrane Database Syst Rev. 2005;1:CD001425.

5. Jemal A, Siegel R, Ward E, et al. Cancer statistics, 2006. CA Cancer J Clin. 2006;56(2):106-130.

6. Chouhan JD, Zamarripa DE, Lai PH, Oramasionwu CU, Grabinski JL. Sunitinib (Sutent): a novel agent for the treatment of metastatic renal cell carcinoma. J Oncol Pharm Pract. 2007;13(1):5-15.

7. Abrams TJ, Lee LB, Murray LJ, Pryer NK, Cherrington JM. SU11248 inhibits KIT and platelet-derived growth factor receptor beta in preclinical models of human small cell lung cancer. Mol Cancer Ther. 2003; 2(5):471-478.

8. Wilhelm SM, Adnane L, Newell P, Villanueva A, Llovet JM, Lynch M. Preclinical overview of sorafenib, a multikinase inhibitor that targets both Raf and VEGF and PDGF receptor tyrosine kinase signaling. Mol Cancer Ther. 2008;7(10):3129-3140.

9. Reddy K. Phase III study of sunitinib malate (SU11248) versus interferon-alpha as first-line treatment in patients with metastatic renal cell carcinoma. Clin Genitourin Cancer. 2006;5(1):23-25.

10. You D, Song SH, Cho YM, et al. Predictive role of tissue-based molecular markers in patients treated with sunitinib for metastatic renal cell carcinoma. World J Urol. 2015;33(1):111-118.

11. Hjortsø MD, Andersen MH. The expression, function and targeting of haem oxygenase-1 in cancer. Curr Cancer Drug Targets. 2014;14(4): 337-347.

12. Was H, Cichon T, Smolarczyk R, et al. Overexpression of heme oxygenase-1 in murine melanoma: increased proliferation and viability of tumor cells, decreased survival of mice. Am J Pathol. 2006;169(6):2181-2198.

13. Nuhn P, Künzli BM, Hennig R, et al. Heme oxygenase-1 and its metabolites affect pancreatic tumor growth in vivo. Mol Cancer. 2009;8:37.

14. Sass G, Leukel P, Schmitz V, et al. Inhibition of heme oxygenase 1 expression by small interfering RNA decreases orthotopic tumor growth in livers of mice. Int J Cancer. 2008;123(6):1269-1277.

15. Balan M, Miery Teran E, Waaga-Gasser AM, et al. Novel roles of c-Met in the survival of renal cancer cells through the regulation of HO-1 and PD-L1 expression. J Biol Chem. 2015;290(13):8110-8120.

OncoTargets and Therapy

\section{Publish your work in this journal}

OncoTargets and Therapy is an international, peer-reviewed, open access journal focusing on the pathological basis of all cancers, potential targets for therapy and treatment protocols employed to improve the management of cancer patients. The journal also focuses on the impact of management programs and new therapeutic agents and protocols on

Submit your manuscript here: http://www.dovepress.com/oncotargets-and-therapy-journal
16. Banerjee P, Basu A, Datta D, Gasser M, Waaga-Gasser AM, Pal S. The heme oxygenase-1 protein is overexpressed in human renal cancer cells following activation of the Ras-Raf-ERK pathway and mediates anti-apoptotic signal. J Biol Chem. 2011;286(38):33580-33590.

17. Was H, Dulak J, Jozkowicz A. Heme oxygenase-1 in tumor biology and therapy. Curr Drug Targets. 2010;11(12):1551-1570.

18. Eisenhauer EA, Therasse P, Bogaerts J, et al. New response evaluation criteria in solid tumours: revised RECIST guideline (version 1.1). Eur J Cancer. 2009;45(2):228-247.

19. Rini BI. Vascular endothelial growth factor-targeted therapy in renal cell carcinoma: current status and future directions. Clin Cancer Res. 2007;13(4):1098-1106.

20. Grandinetti CA, Goldspiel BR. Sorafenib and sunitinib: novel targeted therapies for renal cell cancer. Pharmacotherapy. 2007;27(8):1125-1144.

21. Uchida K, Miyao N, Masumori N, et al. Recurrence of renal cell carcinoma more than 5 years after nephrectomy. Int J Urol. 2002;9(1):19-23.

22. Wysocki PJ. mTOR in renal cell cancer: modulator of tumor biology and therapeutic target. Expert Rev Mol Diagn. 2009;9(3):231-241.

23. Wood LS. New therapeutic strategies for renal cell carcinoma. Urol Nurs. 2010;30(1):40-53.

24. Cohen HT, McGovern FJ. Renal-cell carcinoma. New Engl J Med. 2005;353(23):2477-2490.

25. Klapper JA, Downey SG, Smith FO, et al. High-dose interleukin-2 for the treatment of metastatic renal cell carcinoma: a retrospective analysis of response and survival in patients treated in the surgery branch at the National Cancer Institute between 1986 and 2006. Cancer. 2008; 113(2):293-301.

26. Geertsen PF, Gore ME, Negrier S, Tourani JM, von der Maase H. Safety and efficacy of subcutaneous and continuous intravenous infusion rIL-2 in patients with metastatic renal cell carcinoma. Br J Cancer. 2004;90(6):1156-1162.

27. Hutson TE, Quinn DI. Cytokine therapy: a standard of care for metastatic renal cell carcinoma? Clin Genitourin Cancer. 2005;4(3):181-186.

28. Cho DC. Prognostic biomarkers for patients with advanced renal cell carcinoma treated with VEGF-targeted tyrosine kinase inhibitors. Onco Targets Ther. 2013;6:679-684.

29. Jozkowicz A, Was H, Dulak J. Heme oxygenase-1 in tumors: is it a false friend? Antioxid Redox Signal. 2007;9(12):2099-2117.

30. Escudier B, Eisen T, Stadler WM, et al; TARGET Study Group. Sorafenib in advanced clear-cell renal-cell carcinoma. New Engl J Med. 2007;356(2):125-134.

31. Zimmermann K, Schmittel A, Steiner U, et al. Sunitinib treatment for patients with advanced clear-cell renal-cell carcinoma after progression on sorafenib. Oncology. 2009;76(5):350-354.

32. Motzer RJ, Michaelson MD, Rosenberg J, et al. Sunitinib efficacy against advanced renal cell carcinoma. $J$ Urol. 2007;178(5):1883-1887.

33. Motzer RJ, Hutson TE, Tomczak $P$, et al. Sunitinib versus interferon alfa in metastatic renal-cell carcinoma. New Engl J Med. 2007;356(2):115-124.

34. Motzer RJ, Agarwal N, Beard C, et al. NCCN clinical practice guidelines in oncology: kidney cancer. J Natl Compr Canc Netw. 2009;7(6):618-630.

35. Zhang H, Dong B, Lu JJ, et al. Efficacy of sorafenib on metastatic renal cell carcinoma in Asian patients: results from a multicenter study. BMC Cancer. 2009;9:249.

patient perspectives such as quality of life, adherence and satisfaction The manuscript management system is completely online and includes a very quick and fair peer-review system, which is all easy to use. Visit http://www.dovepress.com/testimonials.php to read real quotes from published authors. 